\title{
Business Cycle Measurement with Semantic Filtering: A Micro Data Approach
}

\author{
Christian Müller* and Eva Köberl ${ }^{\dagger}$
}

16th January 2009

${ }^{*}$ Corresponding author. Zurich University of Applied Sciences, School of Management and ETH Zurich, KOF Swiss Economic Institute, E-mail: much@zhaw.ch

${ }^{\dagger}$ ETH Zurich, KOF Swiss Economic Institute, E-mail: koeberl@kof.ethz.ch 


\begin{abstract}
In this paper we develop a business cycle measure that can be shown to have excellent ex-ante forecasting properties for GDP growth. For identifying business cycle movements, we use a semantic approach. We infer nine different states of the economy directly from firms' responses in business tendency surveys. Hence, we can identify the current state of the economy. We therewith measure business cycle fluctuations. One of the main advantages of our methodology is that it is a structural concept based on shock identification and therefore does not need any - often rather arbitrary - statistical filtering. Futhermore, it is not subject to revisions, it is available in real-time and has a publication lead to official GDP data of at least one quarter. It can therefore be used for one quarter ahead forecasting real GDP growth.
\end{abstract}

JEL classification: E32, C4, C5

Keywords: business cycle measurement, semantic cross validation, shock identification 


\section{Introduction}

Economic theory is very often based on concepts of equilibrium. Market solutions are derived from the idea of intersection of demand and supply, markets clear when the right price is quoted. Likewise, individual decisions such as the choice of optimal inputs in terms of quantity and prices can be modelled by equilibrium approaches where a solution obtains given market structure, profit maximisation objectives and certain state variables. A matter of interest thereby is, how this equilibrium looks in practice. What's this equilibrium like? When and how is it achieved? And how do deviations from this equilibrium which can be interpreted as business cycle fluctuations, look like? These questions are not easy to answer as they depend strongly on the definition of equilibrium.

The literature provides various methods to extract information about business cycle movements. For example, the Hodrick-Prescott-Filter (Hodrick and Prescott, 1997) extracts the difference between trend and cyclical component, which is often interpreted as the business cycle, or the short-lived deviation of actual output from its trend path. There are several other filters such as the Baxter-King-filter (Baxter and King, 1999) available which we may characterise as technical filters. A second branch of business cycle measures use economic theory and econometrics to calculate deviations of actual output from potential output. For doing so, economic theory needs to provide a way for calculating potential output. A natural choice in this case is a hypothetical production function which is then put to the data. Due to its economic underpinning we may call this class of business cycle measures economic filters.

In our approach, we choose yet another way. We use statements of firms about their capacity utilisation on a quarterly frequency and compare these statements to an implicit desired level of capacities. The structure of the data 
allows us to derive a typical dynamic pattern of actual and desired capacity utilisation on a firm level. Based on this pattern and on the semantic content of the particular survey question we are able to define positive, negative shocks and the equilibrium. Owed to the fact that the basis for our identification is a semantic analysis we call this approach semantic filter.

After having extracted the business cycle measure we will compare it to actual GDP growth. We find that our indicator provides excellent ex-ante forecasts for GDP two quarters ahead.

It might be noteworthy that our identification stategy is relatively simple and could be applied to several other countries. Due to its simplicity and robustness with respect to information updates it might be considered as a basis for comparisons of the stance of the business cycles across countries.

The remainder of the paper is structured as follows. In section 2 the framework of the business cycle measurement is described, including details on the data and the empirical methodology used. Section 3 presents the results and performance of the constructed indicator and section 4 concludes.

\section{Business Cycle measurement: framework}

\subsection{The data and its semantic content}

Quite contrary to the usual aggregated analysis we use micro data on the firm level. The data source is the Swiss Economic Institute's (KOF) quarterly business tendency survey in the Swiss manufacturing industry. The data is available from 1999 first quarter to 2007 third quarter and consists of 25119 observations. There are two questions related to capacity utilisation. First, it is asked whether the technical capacities are currently too high, just right or too low (judgment). Secondly, firms are asked to quantify the capacity 
utilisation within the past three months in percentage points, where the firms can choose from a range of $50 \%$ to $110 \%$ in five percentage steps. From the latter we can calculate the percentage change in capacity utilisation from $t$ to $t+1$ and compare this to the judgment about availability of capacities given by the firm in $t$.

The answer to the judgement question is interpreted as follows. A 'too low' is equivalent to a desire for expanding capacities, which should hence result in a reduction of capacity utilisation in the future. Likewise, a 'too high' statement implies the wish for increasing capacity utilisation by lowering capacities, for example.

The key to identify shocks in the economy is our ability to match the qualitative answer which tells whether or not firms are in need of more capacity and the change in their actual capacity utilisation. For example, if firms indicate that their technical capacities are too low and we observe that their use of capacity utilisation increases it is safe to say that this particular firm has been hit by a (positive) shock.

\subsection{Semantic cross validation}

The above interpretation requires some cross-checking with economics. Therefore, we next examine whether or not the data is consistent with basic considerations about plausible firm behaviour 1 The first analysis will be based on contingency tables suggested by Ivaldi (1992). It is constructed as follows (see table 1).

The rows describe the judgment of the firms in $t$ about their current technical capacity; '+' stands for 'too high', '=' for just right, '-' for too low. In

\footnotetext{
1 Borrowing from nonparametric econometrics we label this method semantic cross validation, where economics provides the benchmark for assessing the semantic interpretation.
} 
Table 1: Principle structure of the contingency table

\begin{tabular}{|c||c|c|c|c|}
\hline \multirow{2}{*}{} & \multicolumn{3}{|c|}{ realisation } \\
\cline { 2 - 5 } \multicolumn{2}{|c|}{} & - & $=$ & + \\
\hline \hline \multirow{3}{*}{ judgment } & - & mm & me & mp \\
\cline { 2 - 5 } & $=$ & em & ee & ep \\
\cline { 2 - 5 } & + & pm & pe & pp \\
\hline
\end{tabular}

the columns, the possible outcomes in capacity utilisation changes are listed. A ' + ' means that the level of capacity utilisation has been augmented between $t$ and $t+1, \mathrm{a}^{\text {' }}=$ ' stands for an unchanged level and '-' means a lower level. On the basis of this classification of nine different states of the firms, we are able to identify states that can be associated with either positive or negative shocks. The remaining states will be considered equilibrium situations, or states during which adjustment takes place.

When looking at state $p m$, for example, firms positioned in this field consider their capacities in $t$ as 'too high', but from $t$ to $t+1$ their degree of capacity utilisation still declines. Using the previous arguments we can classify this state as a situation of a negative shock to the particular firm. The argumentation for state $m p$ is similar. As capacities in $t$ are stated as 'too low' and the capacity utilisation rises anyway in the next quarter, we can classify $m p$ as a state of a positive shock. The equilibrium derived from this observations is the state $e e$, where capacity is 'just right' in $t$ and hence there follows no change in capacity utilisation in $t+1$.

Following the same logic $m m$ and $p p$ characterise periods of adjustment towards the desired position, while the interpretation of $m e, p e, e m$, and $e p$ is not that clear cut. Empirically (Müller and Köberl, 2007), it seems that em and $m e$ are very close to the pure equilibrium situation while me and pe lean towards secondary positive and negative shock states. 
For the sample in our study, the repartition of percentage shares to the different states are summarised in table 2

Table 2: Empirical contingency table

\begin{tabular}{|c|c|c|c|c|}
\hline \multirow{2}{*}{\multicolumn{2}{|c|}{$\begin{array}{c}\text { sample } \\
1999-2007\end{array}$}} & \multicolumn{3}{|c|}{ realisation } \\
\hline & & - & $\overline{=}$ & + \\
\hline \multirow{3}{*}{ judgment } & - & 2.7 & 3.0 & 2.4 \\
\hline & $=$ & 25.4 & 30.1 & 25.7 \\
\hline & + & 2.8 & 3.5 & 4.4 \\
\hline \multicolumn{5}{|c|}{$\begin{array}{l}\text { The table entries report the shares of firms } \\
\text { who judge their capacities according to the } \\
\text { row labels and likewise experience a change } \\
\text { in capacity utilisation as indicated by the col- } \\
\text { umn headers. }\end{array}$} \\
\hline
\end{tabular}

The table shows a few interesting features. For example, the majority of firms find itself in a situation where capacities are sufficient (ee). When firms express a desire for more capacities (judgment '-') they increase (realisation '-') their capacities more often than they decrease it (2.7 vs. 2.4). Equivalently, when firms report 'too many' capacities an increase of capacities follows in the next period with the highest probability. By contrast, shocks to this plausible pattern occur not very frequently (positive shock $\mathrm{pm}=2.8$, negative shock $m p=2.4$ ). In a related work (Müller and Köberl, 2007) it has been shown that once being hit by positive shock the typical adjustment path of a firm is $m p \rightarrow m m \rightarrow e p \rightarrow e m \rightarrow e p \ldots$ In other words, after a positive shock firms start to adjust capacities downward $(\mathrm{mm})$ before they enter a period of sustained switching between the near equilibrium states.

All in all we may conclude that the semantic interpretation of the data provided in the previous subsection very well corresponds what is economically plausible. Therefore, we are confident in continuing regrading $m p$ a measure of a positive and $p m$ a measure of a negative shock respectively. 


\subsection{Construction of the indicator}

In this section we describe the calculation of the business cycle measure. We use three approaches which differ only with respect to the way the benchmark is defined. Let $x_{t}$ be either of the nine shares described in table1. For example, in case of a negative shock, $x_{t}=p m_{t}$. Our business cycle measure is given by

$$
b c_{t}^{(i)}=x_{t}-\mu_{t}^{(i)}, \quad \mu_{t}^{(i)}= \begin{cases}\frac{1}{T} \sum_{j=1}^{T} x_{t}, & \text { for } i=1 \\ x_{t}^{*}, & \text { for } i=2 \\ \hat{x}_{t+1} \mid x_{t}, & \text { for } i=3\end{cases}
$$

In case $i=2$ the benchmark is the steady state share of $x_{t}$ obtained from an approximation of the time series process of the nine states (see Müller and Köberl, 2007, for details). The approximation is an ergodic Markov-chain of order one assuming homogenous firms and stationarity. Similarly, for $i=3$ we use the forecast of $x_{t+1}$ based on its past and the estimated Markov-process. Since for $i=1,2$ the benchmark is a constant, the dynamic properties of the resulting business cycle measures are the same. Further, as we are able to distinguish between positive and negative shocks on a semantic basis, the interpretation of positive and negative values of the business cycle indicator changes. In fact, for $i=1,2$ we are going to use $b c_{t}=x_{t}$ directly since it will assume a value of zero in the absence of a shock and the value one if all firms are hit by this shock. In general, positive and negative shocks occur simultaneously, which provides us with a more differentiated picture of the economy as compared to a single measure net of positive and negative shocks.

The choice between $i=3$ and the other two options will be left to the particular purpose of the analysis. In the empirical exercise to follow we focus on $i=1,2$ as it provides a slightly better model fit when estimating quarterly GDP. 


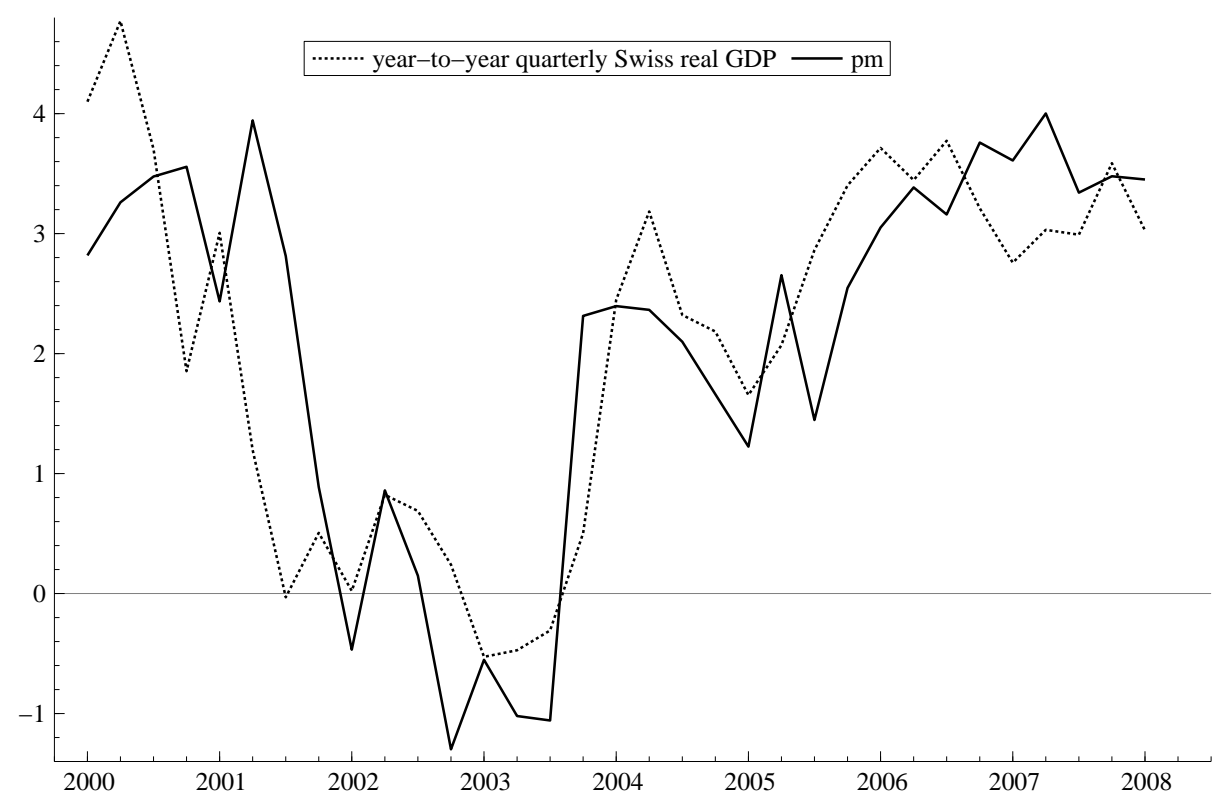

Figure 1: Business cycle measure: inverted negative shock to the economy Source: KOF Quarterly Industry Survey and State Secretariat for Economic Affairs (SECO).

\section{Application}

Before turning to the econometric exercise let us have a look at the business cycle indicator. Figure 1 displays $b c_{t}^{(1,2)}=p m_{t}^{(1,2)}$, that is a negative shock in the economy. The business cylce indicator is range- and mean-adjusted. Furthermore, the figure is scaled to the year-on-year growth rate of quarterly real GDP. To make the picture more accessible, the negative shock has been inverted (multiplied by -1) and plotted against the year-on-year growth rate of quarterly real GDP. By simple visual analysis the correlation between the two series appears pretty high. In fact, the contemporaneous correlation between the GDP growth rate and $b c_{t}$ is -.75 while the correlation with GDP growth one quarter ahead amounts to -.69 . 
Notice that the business cycle indicator is not smoothed or filtered in any way. Therefore, it appears rather spiky in comparison to the filtered GDP growth. Although the noisy appearance may seem inconvenient, it has the big advantage that the release of new data does not invalidate past observations. In other word, by construction, our indicator is free from revisions in the future. Next, we turn to estimation and forecasting GDP growth with the new indicator.

\subsection{Estimating and forecasting GDP growth}

One important desirable property of a business cycle indicator is its ability to track and possibly forecast GDP growth. Our proposal has a publication lead of one quarter. It therefore has the potential of being a good nowcasting tool.

For deriving the most appropriate model we use the following strategy. We first specify a general model for quarterly GDP growth as the dependent variable. The list of exogenous and predetermined variables comprises four lags of quarterly GDP $\left(y_{t}\right)$, the contemporaneous business cycle measure and three of its lags, three seasonal dummies $s$, and a constant. We then let PcGets (see e.g. Hendry and Krolzig, 2004) choose the best model subject to not deleting the constant at any step of the selection procedure. The sample for model selection is 2000 second quarter to 2006 first quarter which admits a valid exante forecasting comparison. The resulting models read (absolute $t$-values in 
parentheses below the coefficient estimates):

$$
\begin{aligned}
\Delta y_{t} & =\underset{(2.62)}{19.54} \mathrm{pm}_{t}-\underset{(1.80)}{13.40} \mathrm{pm}_{t-3}-\underset{(12.90)}{3.48} s_{1, t}-\underset{(6.35)}{1.68} s_{2, t}+\underset{(6.58)}{1.92} \\
\hat{\sigma} & =0.52 \\
\bar{R}^{2} & =0.87
\end{aligned}
$$

The null hypothesis of no autocorrelation up to order four and normality of the residuals cannot be rejected at any conventional level of significance. Hence, the properties of the estimation are very satisfactory and the business cycle indicator appears statistically significant and has the theoretically correct sign.

To complete the application we use equation (3.1) for forecasting. Notice that both model selection and estimation did not include observations after 2006 first quarter. Therefore, we may perform truly ex ante forecasts for the quarters up until 2007 fourth quarter for equation 3.1. In figure 2 the indicator is plotted against the KOF Barometer in multisectoral design and Swiss quarterly real GDP. The forecast is depicted in figure 3

Quite obviously, the forecasting performance is pretty impressive. Not only are the realised values within the $95 \%$ confidence bounds throughout the forecasting period, the absolute deviations are also very small.

Another observation can be made in figure 2 where in addition to quarterly year-to-year growth of real GDP the current official business cycle indicator of $\mathrm{KOF}$ is plotted. The correlation between all three series is rather high. The official series requires considerably more resources for calculation, however, and it is subject to revisions with every new release.

Figure 3 displays the fitted values of the regression. Obviously, the fitted line is much smoother than the original business cycle measure. It therefore of- 


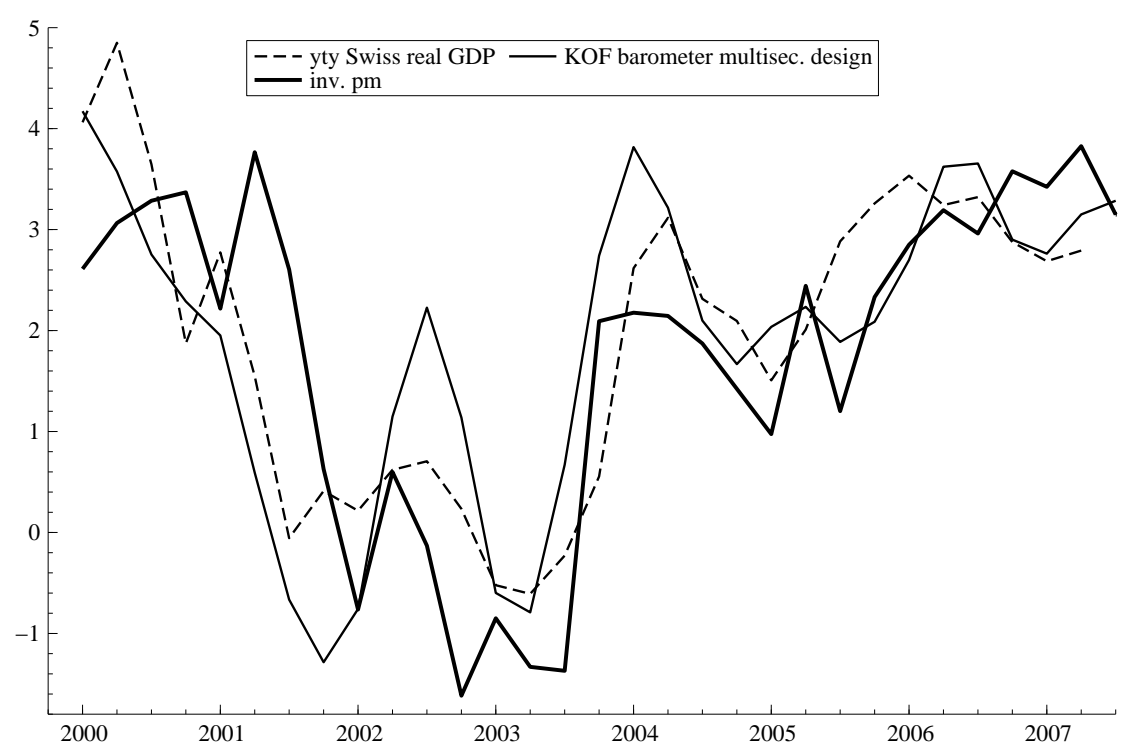

Figure 2: Business cycle measure, KOF Barometer and Swiss quarterly real GDP

Source: KOF Quarterly Industry Survey, KOF Swiss Economic Institute and State Secretariat for Economic Affairs (SECO). 


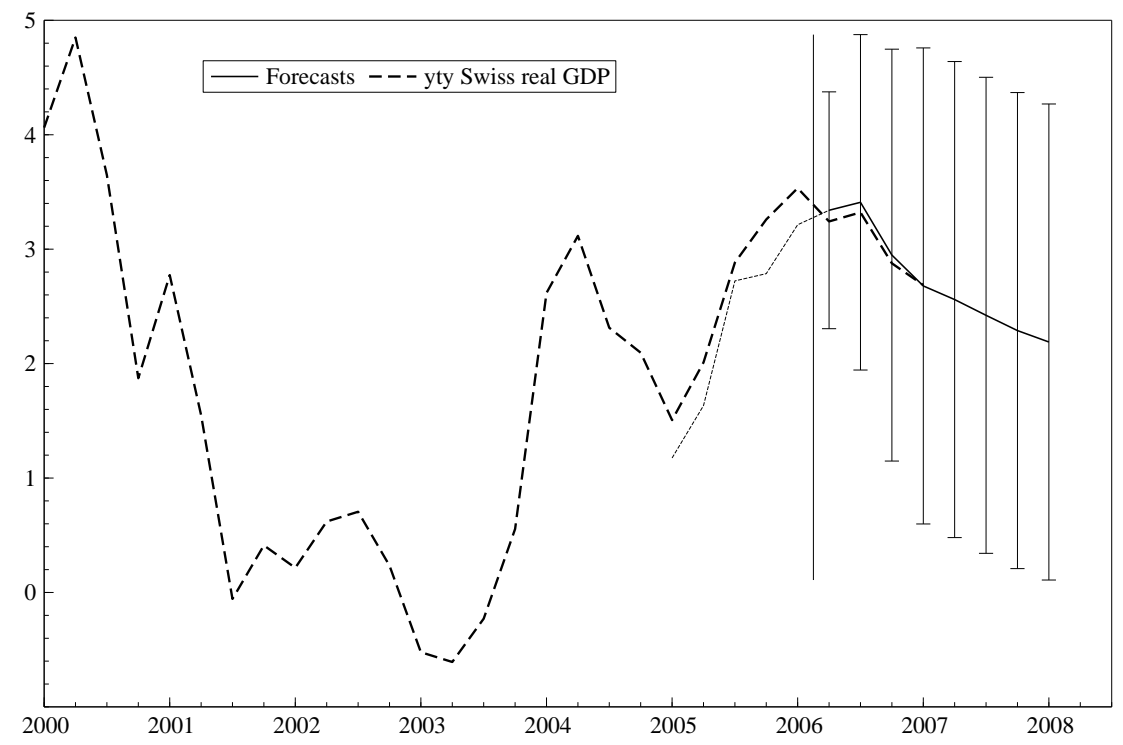

Figure 3: Business cycle measure and forecasting: $b c_{t}=p m_{t}$

fers a possibility to report a more conventional business cycle measure. Doing so would, however, result in revisions in case the estimation is updated with new observations. As yet we have not decided whether or not to accept this disadvantage in exchange for a more traditional, smooth business cycle measure. Notice also that our approach to business cycle measurement is based on identifying shocks. It is not clear that smoothness is a desirable property for shocks.

To conclude this section, we could show that our business cycle indicator does indeed provide valuable information for gauging GDP growth. It evidenced to be a useful tool for nowcasting 2

\footnotetext{
${ }^{2}$ For up-to-date forecasts of Swiss quarterly GDP with this indicator see http://www.s-e-i.ch/Projects/Quantification/BCmeasure/GDPforecasts.htm
} 


\section{Summary and conclusion}

In this paper we describe the derivation of a business cycle indicator that is based on a semantic identification of shocks hitting the economy. We are able to identify positive and negative shocks. We then define the fraction of firms which have been hit by a negative shock as our business cycle measure. Compared to common methods of identifying business cycle movements, our approach has several advantages. First, it is not subject to revisions and has a publication lead of one quarter. Second, the time series has a clear economic interpretation which methods based on statistical filtering have not. Third, it does not require statistical filtering for smoothing. We therefore avoid the assumption of strong priors and implied end point problems or other sources of uncertainty. Fourth, the indicator is very easy to compute in comparison to time-consuming factor analyses or other common methods. Furthermore, it comes along with low marginal costs. The application of our indicator in a simple forecast equation showed that we can provide an adequate nowcasting tool that can be used for one quarter ahead forecasting real GDP growth. 


\section{References}

Baxter, M. and King, R. (1999). Measuring business cycles: Approximate band-pass filters for economic time series, Review of Economics and Statistics 81: 573-593.

Hendry, D. F. and Krolzig, H. (2004). We ran one regression, Oxford Bulletin of Economics and Statistics 66(5): 799 - 810.

Hodrick, R. and Prescott, E. C. (1997). Postwar U.S. Business Cycles: An Empirical Investigation, Journal of Money, Credit, and Banking 29(1): 1 $-16$.

Ivaldi, M. (1992). Survey Evidence on the Rationality of Expectations, Journal of Applied Econometrics 7(3): 225 - 41.

Müller, C. and Köberl, E. (2007). The speed of adjustment to demand shocks: A Markov-chain measurement using micro panel data, Working Paper 170, KOF Swiss Economic Institute at the Swiss Federal Institute of Technology Zurich. http://www.ssrn. com/abstract=998019. 